\title{
Measurement of Rotational Temperature in a Molecular Beam with Femtosecond Laser Pulses
}

\author{
Kazumichi YOSHII, Godai MIYAJI and Kenzo MIYAZAKI \\ Advanced Laser Science Research Section, Institute of Advanced Energy, Kyoto University, Kyoto \\ 611-0011, Japan \\ E-mail: miyazaki@iae.kyoto-u.ac.jp
}

\begin{abstract}
We apply high-order harmonic generation to sensitive measurements of molecular rotational temperature in a thin supersonic gas beam. The method uses nonresonant pump and probe femtosecond laser pulses to generate the harmonic radiation from coherently rotating molecules. The rotational temperature of molecules can accurately be derived with high spatial and temporal resolutions from the Fourier spectrum of time-dependent signals. The validity of method was tested for an expanding flow of $\mathrm{N}_{2}$ beam with a rapid temperature decrease. The results show the versatile applicability of this method.

DOI:10.2961/jlmn.2010.02.0004
\end{abstract}

Keywords: rotational temperature, supersonic molecular beam, rotational coherence spectroscopy, high-order harmonic generation, femtosecond laser

\section{Introduction}

Supersonic molecular beams have been used extensively in material processing, as well as in a variety of fields of molecular science and technology. Major advantages of a supersonic beam are associated with its ability to generate an isolated ensemble of collision-free molecules with extremely low temperature and make microscopic experiments possible [1]. In the experiment using a molecular beam, the rotational temperature $T_{\text {rot }}$ is one of the most important parameters to characterize the molecular system concerned. The most powerful tool to measure $T_{\text {rot }}$ in a thin supersonic beam has been coherent anti-Stokes Raman scattering (CARS), where tunable pulsed lasers are usually used to observe the spectrum in resonance to the rotational levels $[2,3]$. The development of spectroscopic methods using CARS has greatly improved the accuracy of $T_{\text {rot }}$ measurement to study detailed rotational dynamics in a pulsed molecular beam. In addition to the frequency domain measurement, the time-domain CARS spectroscopy has also been developed using femtosecond (fs) laser pulses [4,5], which has the advantage of high temporal resolution. However, the CARS spectroscopy, as well as its related nonlinear optical methods such as degenerate four-wave mixing (DFWM), often meets some difficulties arising from the complexity of molecular rotational transitions, e.g., nonresonant background and signal frequencies close to those of the pump laser pulses $[5,6]$.

In this paper we report a sensitive and versatile method to measure $T_{\text {rot }}$ in a supersonic molecular beam, which has high spatial and temporal resolutions in a broad experimental range of molecular beams. The method employs a pump and probe technique with fs laser pulses, where the pump pulse creates a rotational wave packet that leads to temporal alignment of molecules, and the probe pulse generates high-order harmonic radiation from aligning molecules $[7,8]$. In contrast to CARS spectroscopy and DFWM, the present method detects the harmonic radiation in a vac- uum ultraviolet spectral region far from the fundamental wavelength concerned. The harmonic signal measured as a function of time delay $\Delta t$ between the pump and probe pulses provides us definite information of $T_{\text {rot }}$ in the supersonic molecular beam.

\section{Experimental}

The experimental setup was the same as in our previous experiments [7-9]. Briefly, the linearly polarized, 40-fs, $800 \mathrm{~nm}$, pump and probe pulses with a time delay $\Delta t$ are produced from the output of a Ti:sapphire laser system. These pulses were collinearly focused with a $500 \mathrm{~mm}$ focal-length lens into a pulsed $\mathrm{N}_{2}$ beam, where the spot size was measured to be $70 \mu \mathrm{m}$ with a CCD camera through an imaging optics, together with its spatial intensity distribution. The pump pulse induces transient alignment of molecules through the formation of a rotational wave packet, which is recurrent under field free conditions, and the delayed probe pulse generates high harmonic radiation from coherently rotating molecules as a function of $\Delta t$. The pump and probe intensities were optimized for the measurement of $T_{\text {rot }}$ in the $\mathrm{N}_{2}$ beam and fixed at 0.75 and $2.2 \times$ $10^{14} \mathrm{~W} / \mathrm{cm}^{2}$, respectively. The harmonic radiation produced was detected by an electron multiplier mounted on a vacuum ultraviolet monochromator.

The laser intensity in the interaction region was carefully evaluated, since $T_{\text {rot }}$ to be derived is dependent of the pump intensity, as well as the pulse width.

The pulsed supersonic $\mathrm{N}_{2}$ beam of $160 \mu$ s duration is jetted into vacuum from the 1-mm diameter nozzle of a piezoelectric valve. The pulsed valve was synchronously operated with the fs laser at a repetition rate of $10 \mathrm{~Hz}$. The source pressure $P_{0}$ in the valve was changed in a range from 1 to $9 \mathrm{~atm}$ at room temperature. The pulsed valve attached on a vacuum chamber was mounted on a positioning stage, and the distance $x$ between the nozzle exit and 
the optic axis was changed by translating the stage (see the inset in Fig. 5).

\section{Theory}

In the present study we assume that the rotational distribution in the gas jet was in a thermal equilibrium with a Boltzmann distribution. Then the recently developed theory provides the $n$-th harmonic signal per molecule as a function of $\Delta t[10,11]$. The validity of theoretical expressions for $\mathrm{N}_{2}$ and $\mathrm{O}_{2}$ has been verified with our recent experimental study [9]. For $\mathrm{N}_{2}$ used in this work, the harmonic signal is given as

$$
\begin{aligned}
S^{(n)}(\Delta t) & \sim c_{1}+c_{2}<<\cos ^{2} \theta>>(\Delta t)+c_{3}<<\cos ^{2} \theta>^{2}>(\Delta t) \\
& +c_{4}<<\cos ^{4} \theta>>(\Delta t)+---
\end{aligned}
$$

where $c_{\mathrm{i}}$ is the coefficient depending on the intensity and duration of the probe pulse $[10,11]$. The inner bracket of $<<\cos ^{2} \theta>>$ and subsequent terms stands for the expectation value for the rotational wavepacket consisting of coherently populated rotational states at $\Delta t=0$, and the outer bracket corresponds to the thermal average over the Boltzmann distribution at $T_{\text {rot }}$. It is noted that the timedependent harmonic signal $S^{(n)}$ is dominated by the initial rotational temperature $T_{\text {rot }}$ included in such thermally averaged terms as $<<\cos ^{2} \theta>>$, once the rotational wave packet
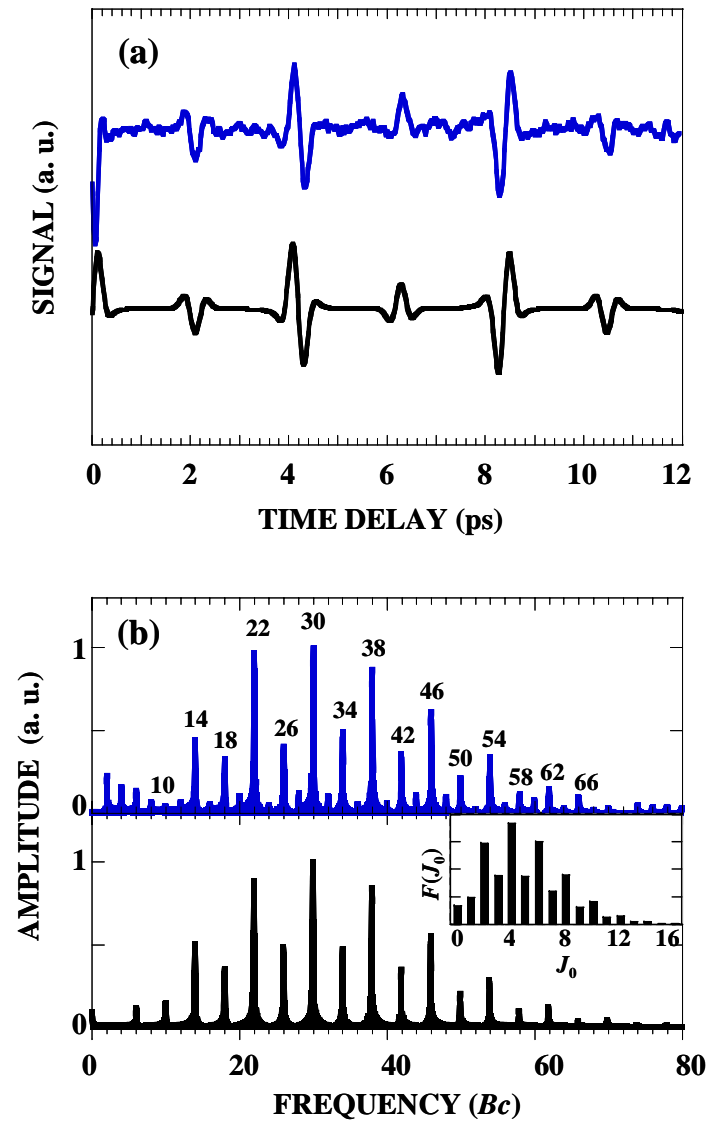

Fig. 1 (a) Time-dependent 19th harmonic signals observed at $x / D=0.1$ for $P_{0}=1 \mathrm{~atm}$ (upper) and calculated (lower) for $T_{\text {rot }}=110 \mathrm{~K}$, and (b) their frequency spectra. The inset in (b) indicates the rotational distribution at $T_{\text {rot }}=110 \mathrm{~K}$. The pump and probe intensities used in the experiment and calculation are 0.75 and $2.2 \times 10^{14} \mathrm{~W} / \mathrm{cm}^{2}$, respectively. is formed at $\Delta t \approx 0$ with the pump pulse. The evaluation of $T_{\text {rot }}$ in the experiment can accurately be made by comparing the frequency spectrum of the observed time-dependent harmonic signal with the theoretical, because the spectrum is very sensitive to the distribution of rotational states at the unique fitting parameter $T_{\text {rot }}$, as shown below.

\section{Results and Discussions}

Figure 1 shows (a) typical examples of the timedependent harmonic signal observed at the relative position $x / D=0.1$ for $P_{0}=1$ atm (upper trace) and the signal $\phi(\Delta t)$ calculated for the same pump and probe intensities (lower trace), and (b) their corresponding frequency spectra [12]. In the calculation for $\mathrm{N}_{2}$, we used only the single dominant term $\phi(\Delta t) \equiv c_{2}<<\cos ^{2} \theta>>(\Delta t)$ to reproduce the signal and its frequency spectrum, since the other terms provide the identical rotational distribution to derive $T_{\text {rot }}$ [9]. In Fig. 1, the best agreement between theory and experiment is obtained for $T_{\text {rot }}=110 \mathrm{~K}$. As discussed in detail in the previous studies $[8,10]$, the time-dependent behavior of harmonic signal is dominated by the beat frequency $\Omega \equiv\left(E_{J+2}\right.$ $\left.-E_{J}\right) / 2 \pi=(4 J+6) B c$ between any pair of rotational states populated through the Raman transition $\Delta J= \pm 2$, where $E_{J}$ is the eigenenergy of a state with the angular momentum $J$, $B$ is the rotational constant, and $c$ is the speed of light. The frequency spectrum represents a distribution of coherently
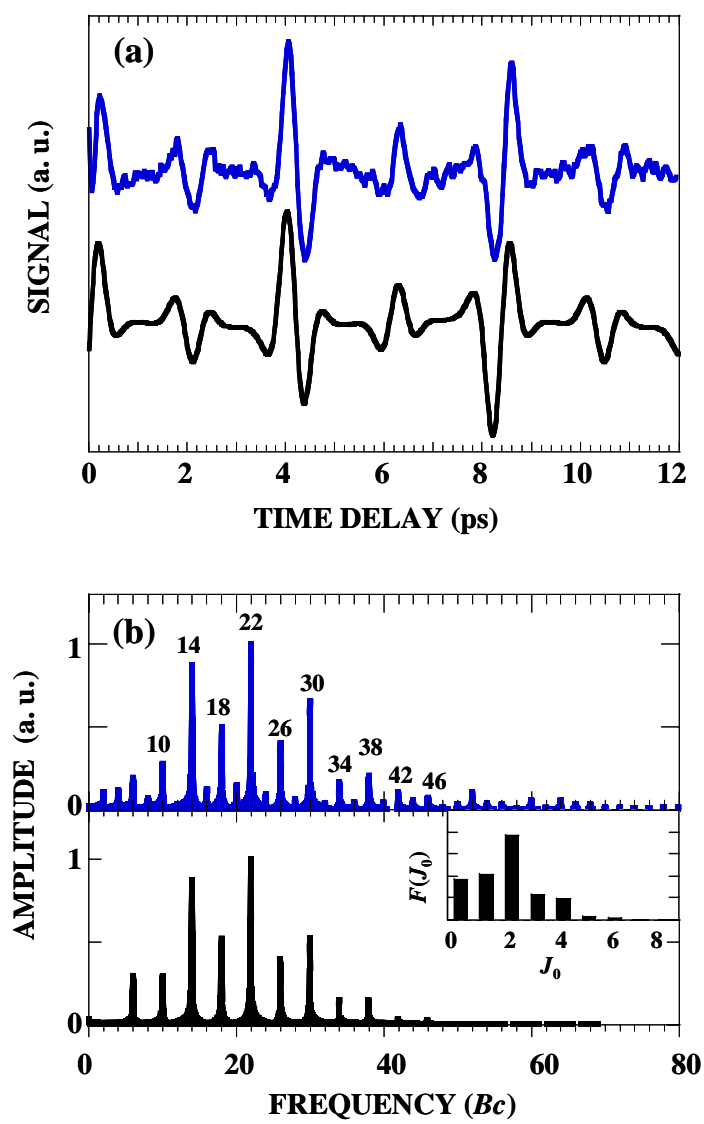

Fig. 2 (a) Time-dependent 19th harmonic signals observed at $x / D=1.5$ for $P_{0}=9 \mathrm{~atm}$ (upper) and calculated (lower) for $T_{\text {rot }}=20 \mathrm{~K}$, and (b) their frequency spectra. The inset indicates the rotational state distribution at $T_{\text {rot }}=20 \mathrm{~K}$. The pump and probe intensities are the same as in Fig. 1. 
rotating molecules, and their coherent superposition produces the characteristic time-dependent signal including the revival structure of molecular alignment. The inset in the lower spectrum of Fig. 1(b) represents the initial rotational distribution at $T_{\text {rot }}=110 \mathrm{~K}$. Since $\mathrm{N}_{2}$ is a homonuclear molecule with nuclear spin of 1 , the population $F\left(J_{0}\right)$ of even and odd $-J_{0}$ states has a ratio of $2: 1$. This population alternation is clearly seen in the distribution of $\Omega$. As shown in the inset in Fig. 1(b), the initial population is peaked at $J=4$. This population peak is shifted to the $J=6$ state for $\Omega=30 B c$ with the pump pulse. The experimental result shown in Fig. 1(b) includes the additional small frequency components that originate from the higher terms in $S^{(n)}$, as discussed in our previous studies $[8,10]$.

The frequency spectrum depends on only $T_{\text {rot }}$, when the pump and probe intensities are fixed for a molecular species. To see the sensitivity of the frequency distribution to $T_{\text {rot }}$, the time-dependent 19 th harmonic signal was observed at a different position $x / D=1.5$ for a higher pressure of $P_{0}=9 \mathrm{~atm}$, where the jetted molecules were expected to have a much lower rotational temperature. Figure 2 shows the results of (a) the observed (upper) and calculated (lower) time-dependent harmonic signals and (b) their frequency spectra, where the best agreement between theory and experiment is obtained for a rotational temperature of $T_{\text {rot }}=20 \mathrm{~K}$. The frequency spectrum represents the peak amplitude at $J=4$ with $\Omega=22 B C$ in this distribution. It is clear that the shift from $\Omega=30 B c$ in Fig. 1(b) to $22 B c$ for the peak amplitude is due to a decrease from $T_{\text {rot }}=110 \mathrm{~K}$ to $20 \mathrm{~K}$ and resulting relative increase in the initial populations of lower rotational states, as shown in the inset of Fig. 2(b).

The above result is consistent with the well-known

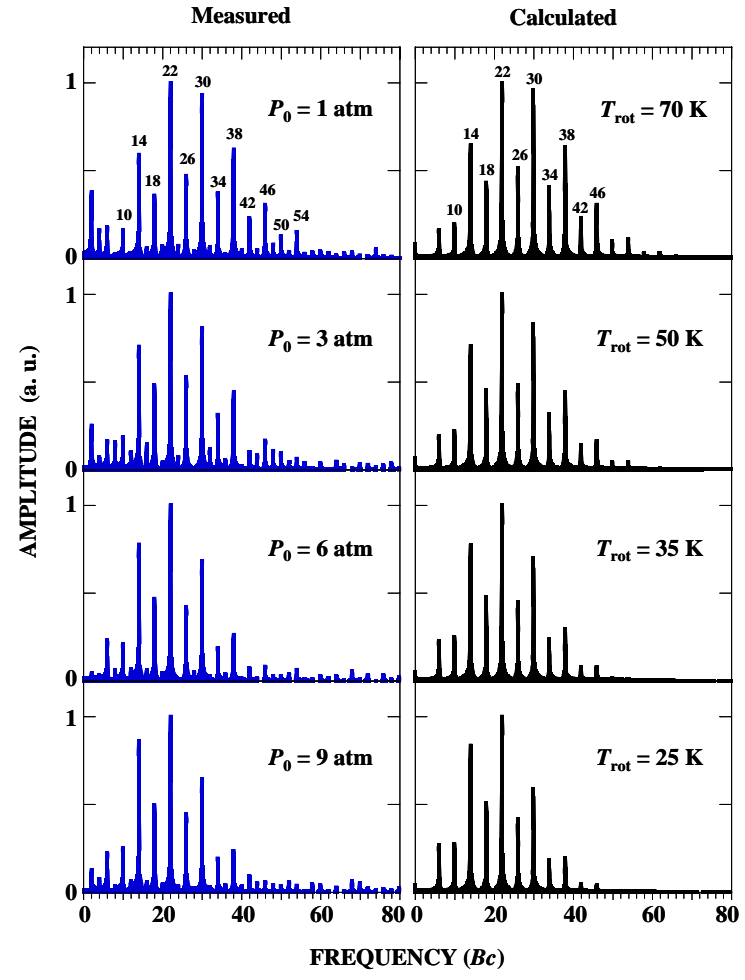

Fig. 3 The frequency spectra of the 19th harmonic signals measured for various $P_{0}$ at $x / D=0.5$ (left row) and calculated (right row). fact that $T_{\text {rot }}$ in the free expansion of a supersonic molecular beam decreases with an increase in $x / D$ and/or $P_{0}[13,14]$. In particular, the decrease in $T_{\text {rot }}$ is so rapid in a region of non-isentropic gas expansion, which is usually observed for a small value of $x / D$ [14]. To see this and confirm the applicability of the present method, we measured $T_{\text {rot }}$ as a function of $x / D(<2)$ for different $P_{0}$, using the 19th harmonic signals. Figure 3 shows the frequency spectra measured for various $P_{0}$ at $x / D=0.5$ (left row) and those calculated (right row). The spectra measured at various $x / D$ for $P_{0}=6$ atm and calculated are shown in Fig. 4. In the figures we also show the $T_{\text {rot }}$ on which the calculations are based.

The result of $T_{\text {rot }}$ measured is plotted as a function of $x / D$ as shown in Fig. 5. As expected, a rapid decrease in $T_{\text {rot }}$ is observed in the region of $x / D=0.1-1.0$, e.g., $T_{\text {rot }}=110 \mathrm{~K}$ decreases to $50 \mathrm{~K}$ at $P_{0}=1 \mathrm{~atm}$. For comparison, we calculated the translational temperature $T$ in the same region of $x / D$, assuming the isentropic expansion of molecular gas [14], which is usually less than $T_{\text {rot }}$. The calculation provided $T=245 \mathrm{~K}$ at $x / D=0.1$ and its monotonous decrease to $T=94 \mathrm{~K}$ at $x / D=1.5$. As shown in Fig. 5, the measured value of $T_{\text {rot }}$ is much lower than the calculated value of $T$. This characteristic property of $T_{\text {rot }}$ in a region of small $x / D$ reconciles with those reported so far for the pulsed supersonic free jets of $\mathrm{N}_{2}$ [15] and other molecules [16,17].

Throughout the present measurement, as described above, the pump intensity was fixed at $0.75 \times 10^{14} \mathrm{~W} / \mathrm{cm}^{2}$. The accuracy of $T_{\text {rot }}$ obtained in this measurement might be estimated by assuming a different value of the intensity. The result has shown that a change of $10 \%$ in the intensity produces a shift of $10-15 \mathrm{~K}$ in $T_{\text {rot }}$.

The present technique provides a versatile way to meas-

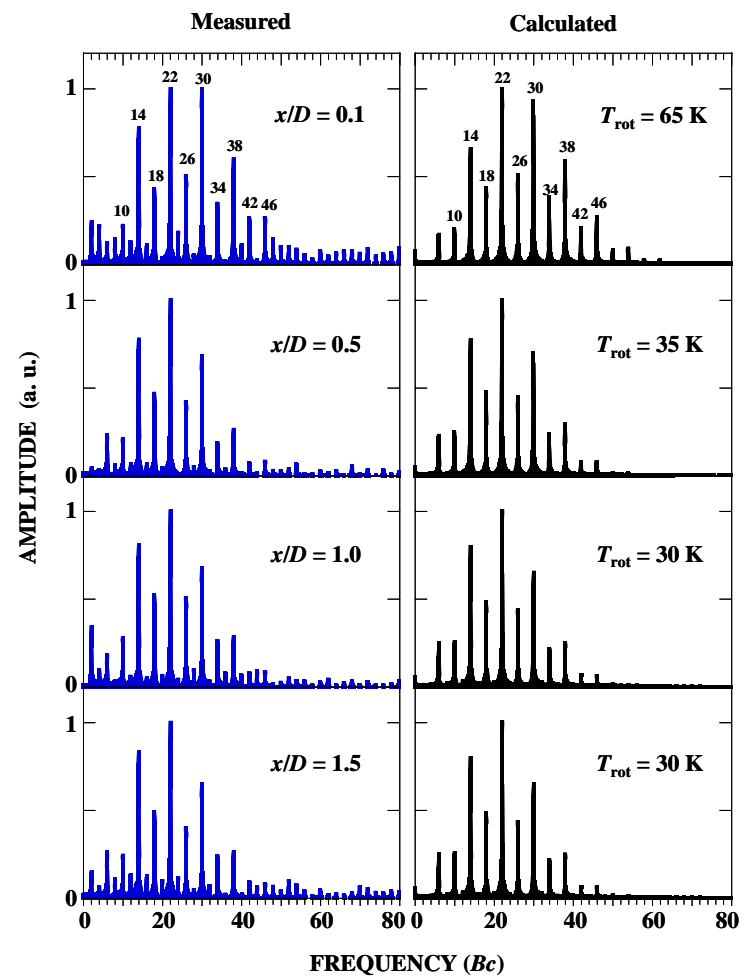

Fig. 4 The frequency spectra of the 19th harmonic signals measured for $P_{0}=6 \mathrm{~atm}$ at various $x / D$ (left row) and calculated (right row). 


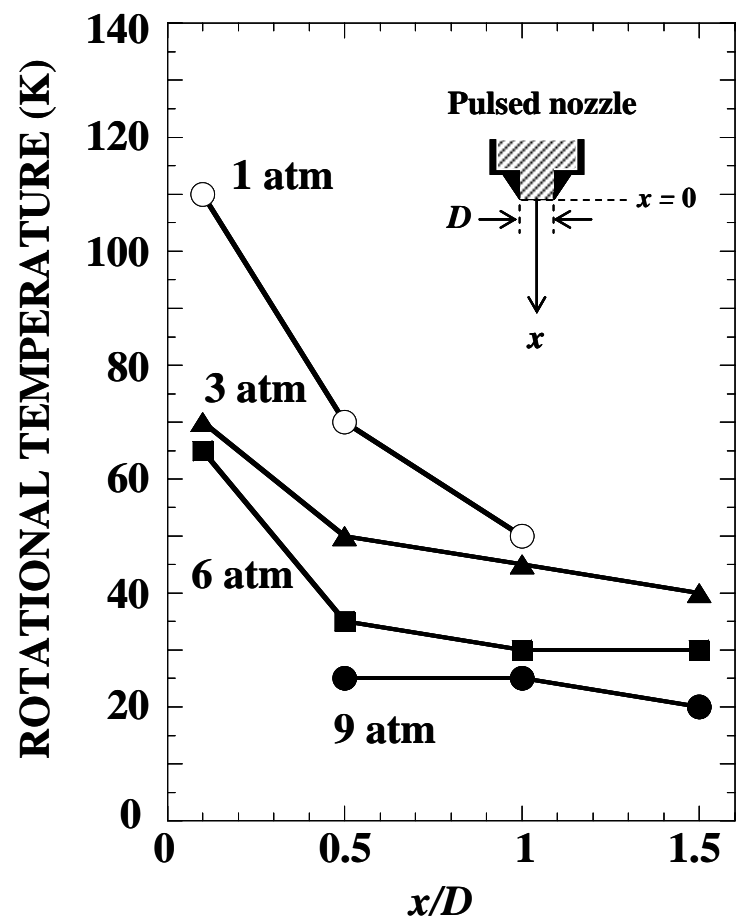

Fig. 5 Rotational temperature in the supersonic $\mathrm{N}_{2}$ gas jet measured as a function of the distance $x / D$ from the nozzle for different source pressures.

ure $T_{\text {rot }}$, since the nonresonant fs laser can commonly be applied to different kinds of molecules under a variety of conditions for HHG, and the simulation of harmonic signals should be possible for any kind of molecules according to the theory $[10,11]$. In fact we confirmed that $T_{\text {rot }}$ in pulsed $\mathrm{O}_{2}$ and $\mathrm{CO}_{2}$ supersonic beams could accurately be estimated with this method. The temporal resolution in the measurement is on a ps scale with a spatial resolution in the focused beam size. In addition, the time domain measurement has allowed us to evaluate $T_{\text {rot }}$ of an ensemble of lowdensity molecules by observing multiphoton ionization of aligned molecules with a time-of-flight mass spectrometer [18]. The results obtained will be presented and discussed elsewhere.

\section{Summary}

In summary, we have demonstrated a sensitive and versatile method to measure rotational temperature in a pulsed supersonic molecular beam, which is based on the observa- tion of time-dependent HHG signal from coherently rotating molecules. The results obtained are consistent with those reported so far.

\section{Acknowledgment}

The authors thank Abdurrouf for helpful discussion. This work is partially supported by the Grant-in-Aid for Scientific Research (A) 18206010.

\section{References}

[1] See, e.g., Atomic and molecular beam methods, G. Scoles, ed. (Oxford Univ., New York, 1988).

[2] P. Huber-Wälchli, D. M. Guthals, and J. W. Nibler, Chem. Phys. Lett. 67, 233-236 (1979).

[3] M. D. Duncan, P. Österlin, and R. L. Byer, Opt. Lett. 6, 90-92 (1981).

[4] T. Lang, K. -L. Kompa, and M. Motzkus, Chem. Phys. Lett. 310, 65-72 (1999).

[5] T. Lang, M. Motzkus, H. M. Frey, and P. Beaud, J. Chem. Phys. 115, 5418-5426 (2001).

[6] T. Hornung, H. Skenderović, K. -L. Kompa, and M. Motzkus, J. Raman Spectrosc. 35, 934-938 (2004).

[7] M. Kaku, K. Masuda, and K. Miyazaki, Jpn. J. Appl. Phys. 43, L591- L593 (2004).

[8] K. Miyazaki, M. Kaku, G. Miyaji, A. Abdurrouf, and F. H. M. Faisal, Phys. Rev. Lett. 95, 243903 (2005).

[9] K. Yoshii, G. Miyaji, and K. Miyazaki, Phys. Rev. Lett. 101, 183902 (2008).

[10] F. H. M. Faisal, A. Abdurrouf, K. Miyazaki, and G. Miyaji, Phys. Rev. Lett. 98, 143001 (2007).

[11] A. Abdurrouf and F. H. M. Faisal, Phys. Rev. A 79, 023405 (2009).

[12] In producing the frequency spectrum of the observed time-dependent signal, we ignore the initial rapid change at $\Delta t \approx 0$ that is induced by the high intensity of superimposed pump and probe pulses [8].

[13] R. J. Gallagher, and J. B. Fenn, J. Chem. Phys. 60, 3487-3491 (1974).

[14] D. R. Miller, in ref. 1, pp.14-53.

[15] H. -D. Barth, F. Huisken, and A. A. Ilyukhin, Appl. Phys. B 52, 84-89 (1991).

[16] H. -D. Barth, and F. Huisken, Chem. Phys. Lett. 169, 198-203 (1990).

[17] F. Huisken, and T. Pertsch, Appl. Phys. B 41, 173-178 (1986).

[18] K. Miyazaki, T. Shimizu, and D. Normand, J. Phys. B 37, 753-761 (2004)

(Received: July 10, 2009, Accepted: March 15, 2010) 